\title{
Systemic Delivery of siRNA Specific for Silencing TLR4 Gene Expression Reduces Diabetic Cardiomyopathy in a Mouse Model of Streptozotocin-Induced Type 1 Diabetes
}

\author{
Yuwei Zhang $\cdot$ Yang Li $\cdot$ Xuefang Huang $\cdot$ Fang Zhang $\cdot$ \\ Lizhi Tang $\cdot$ Shishi Xu $\cdot$ Yuqi Liu $\cdot$ Nanwei Tong $\cdot$ Weiping Min
}

Received: February 7, 2020 / Published online: April 13, 2020

(C) The Author(s) 2020

\begin{abstract}
Introduction: Diabetic cardiomyopathy is a cardiac dysfunction in patients with diabetes which may lead to overt heart failure and death. Toll-like receptor (TLR) signaling triggers diabetic cardiomyopathy through various mechanisms, one of which is the upregulation of TLR4 expression. The aim of this study was to delineate the role of TLR4 in diabetic cardiomyopathy.

Methods: C57BL/6 mice were injected with streptozotocin to induce diabetes. The experimental and control groups were treated with $5 \mu \mathrm{g}$ of TLR4 small interfering RNA (siRNA) or scrambled siRNA. Cardiac histopathology was
\end{abstract}

Digital Features To view digital features for this article go to https://doi.org/10.6084/m9.figshare.11973750.

Y. Zhang $\cdot$ Y. Li $\cdot$ F. Zhang $\cdot$ L. Tang $\cdot$ S. Xu ·

Y. Liu $\cdot$ N. Tong $(\bowtie)$

Department of Endocrinology and Metabolism,

West China Hospital, Sichuan University, Sichuan,

China

e-mail: buddyjun@hotmail.com

W. Min $(\varangle)$

Departments of Surgery, Pathology, Medicine, Oncology, University of Western Ontario, London, ON, Canada

e-mail: mweiping@uwo.ca

X. Huang

Endocrine and Metabolic Center, People's Hospital of Karamay, Xinjiang, China evaluated by hematoxylin and eosin, Sirius red, and immunofluorescence staining after treatment with TLR4 siRNA. The myocardial fibrosis and inflammatory factors were detected by quantitative real-time polymerase chain reaction after treatment with TLR4 siRNA. The myocardial function was evaluated by echocardiography after treatment with TLR4 siRNA.

Results: Compared with non-diabetic mouse hearts, hypertrophy, fibrosis, inflammation of cardiomyocytes, and myocardial dysfunction were significantly increased in diabetic mice $(p<0.05)$. Knockdown of TLR4 decreased hypertrophy, fibrosis, inflammation of cardiomyocytes, and myocardial dysfunction $(p<0.05)$. Cardiomyocytic cross-sectional areas in hearts of TLR4 siRNA-treated diabetic mice were similar to those of the sham-treated mice $(p>0.05)$. The induction of expression of cardiac fetal genes, beta-myosin heavy chain ( $\beta$ MHC) and atrial natriuretic peptide (ANP), which are two markers of cardiac hypertrophy, was significantly reduced in TLR4 siRNA-treated hearts compared with controls $(p<0.05)$. Moreover, siRNA-mediated silencing of TLR4 reduced diabetes-induced collagen deposition $(p<0.05)$. Paralleled with changes in collagen deposition and the expression of collagen I and collagen III, knockdown of TLR4 also reduced the expression of transforming growth factor- $\beta 1$ (TGF $\beta 1)$ mRNA $(p<0.05)$. The increased expression of intercellular cell adhesion molecule 1 (ICAM-1) and vascular cell adhesion 
molecule 1 (VCAM-1) was significantly attenuated by TLR4 siRNA treatment in the hearts of diabetic mice $(p<0.05)$. Furthermore, both fractional shortening (FS) and ejection fraction (EF) values were preserved in TLR4 siRNA-treated diabetic mice compared with control siRNAtreated mice $\quad(31.80 \% \pm 2.82 \% \quad$ vs. $28.50 \% \pm 5.83 \% \quad$ for $\quad \mathrm{FS}, \quad p<0.05)$ $(57.95 \% \pm 6.48 \%$ vs. $45.34 \% \pm 4.25 \%$ for $\mathrm{EF}$, $p<0.05)$.

Conclusion: Our study used siRNA to specifically silence TLR4 gene expression in the diabetic mouse heart in vivo and to investigate the role that TLR4 plays in diabetic cardiomyopathy. It is likely that silencing of the TLR4 gene through siRNA could prevent the development of diabetic cardiomyopathy.

Keywords: Diabetic cardiomyopathy; Mouse model; Systemic delivery of siRNA; TLR4; Type 1 diabetes

\section{Key Summary Points}

Why carry out this study?

Diabetic cardiomyopathy is a cardiac dysfunction in patients with diabetes which may lead to overt heart failure and death. Toll-like receptor (TLR) signaling triggers diabetic cardiomyopathy through various mechanisms, one of which is the upregulation of TLR4 expression

The aim of this study was to delineate the role of TLR4 in diabetic cardiomyopathy

\section{What was learned from the study?}

Our study used siRNA to specifically silence TLR4 gene expression in the diabetic mouse heart in vivo and to investigate the role that TLR4 plays in diabetic cardiomyopathy

It is likely that silencing of the TLR4 gene through siRNA could prevent the development of diabetic cardiomyopathy

\section{INTRODUCTION}

Diabetic cardiomyopathy was first proposed in 1972 by Rubler and is a disorder of the heart muscle in patients with diabetes $[1,2]$. It can result in an insufficiency of general blood circulation, reaching a state called heart failure, along with pulmonary or peripheral edemas [3-5]. Cardiomyopathy may occur throughout diabetes, and its major clinical manifestations are hypertrophy, fibrosis, inflammation of cardiomyocytes, and myocardial dysfunction. There might be a close relationship between hypertrophy, fibrosis, inflammation of cardiomyocytes, myocardial dysfunction, and ventricular remodeling $[6,7]$. At present, there is no specific method to diagnose diabetic cardiomyopathy. The main problem to be resolved is how to delay and prevent the development of this heart disease $[8,9]$.

Toll-like receptor 4 (TLR4), a proximal signaling receptor in innate immune responses to lipopolysaccharide (LPS) of Gram-negative pathogens, is expressed in the heart and vasculature. TLR4 binds to LPS from the Gram-negative bacteria and autogenous ligands, such as heat shock proteins and fibronectin, which are released during oxidative stress. Increased TLR4 expression has been observed in patients with heart failure and in ischemic hearts [10-13].

Apoptosis of cardiomyocytes contributes to diabetic cardiomyopathy [14]. Recently, we have shown that cardiomyocytic apoptosis is mediated through the TLR4-dependent pathway under diabetic conditions [15]. The current study was undertaken to investigate whether systemic delivery of siRNA specific for gene silencing of TLR4 would reduce cardiomyopathic changes in a type 1 diabetic mouse model.

\section{METHODS}

\section{Animals}

Male adult C57BL/6 mice, aged from 6 to 8 weeks, were purchased from the Jackson Laboratory (Bar Harbor ME, USA). All mice were anesthetized through injection in the 
abdominal cavity (1\% pentobarbital sodium, $80 \mathrm{mg} / \mathrm{kg}$ ), they were killed by carbon dioxide inhalation of $\mathrm{CO}_{2}$ at a flow rate of more than $30 \%$ of the chamber volume per minute, and death was confirmed by cervical dislocation. The were incinerated after the experiment. All experimental procedures were approved by the Animal Use Subcommittee at Sichuan University.

\section{siRNA Expression Vectors}

Three target sequences of the TLR4 gene were selected. The oligonucleotides containing sequences specific for TLR4 (5'-GATCCCGTAT TAGGAACTACCTCTATGCTTGATATCCGGCA TAGAGGTAGTTCCTAATATTTTTTCCAAA-3' and $5^{\prime}$-AGCTTTTGGAAAAAATATTAGGAACTA CCTCTATGCCGGATATCAAGCATAGAGGTAG TTCCTAATACGG-3'; 5'-GATCCCGTTGAAAC TGCAATCAAGAGTGTTGATATCCGCACTCTTG ATTGCAGTTTCAATTTTTTCCAAA- $3^{\prime}$ and $5^{\prime}$-AG CTTTTGGAAAAAATTGAAACTGCAATCAAGAG TGCGGATATCAACACTCTTGATTGCAGTTTCA ACGG-3'; 5'-GATCCCATTCGCCAAGCAATGGA ACTTGATATCCGGTTCCATTGCTTGGCGAATT TTTTTCCAAA- ${ }^{\prime}$ and 5'-AGCTTTTGGAAAAA AATTCGCCAAGCAATGGAACCGGATATCAAG TTCCATTGCTTGGCGAATGG-3') were synthesized and annealed.

\section{TLR4 siRNA Expression Vector Construction}

A TLR4 siRNA expression vector was constructed to express hairpin shRNA, which was demonstrated in detail in our previous study [15].

\section{Hyperglycemic Mouse Model}

Low dose streptozotocin (STZ) can maintain insulin secretory function and produce a diabetes state. The diabetes state models the partial and continuous loss of $\beta$ cells in type 2 diabetes mellitus. The metabolic characteristics of STZinduced diabetic mouse induced by low dose STZ are closer to those of type 2 diabetes in humans compared with the gene rodent models
$[16,17]$. So, in the study, we chose low dose STZ for type 2 diabetes induction. Consecutive peritoneal injections of streptozotocin (STZ) $(50 \mathrm{mg} / \mathrm{kg} /$ day) were utilized to induce diabetes in 14 2-month-old adult male mice for each group. Whole blood was gathered from the mouse tail vein $72 \mathrm{~h}$ after the last STZ injection. The data of random glucose levels were measured through the One Touch Ultra 2 blood glucose monitoring system (Life Scan Inc, CA, USA). The mice would be used for the study only if they were considered diabetic and had hyperglycemia (fasting blood glucose at least $16.7 \mathrm{mM}$ ) at $72 \mathrm{~h}$ after the STZ injections [18]. Citrate buffer-treated mice were used as the non-diabetic control. Two months after the induction of diabetes, all the mice were subjected to the following experiments.

\section{Treatment of TLR4 siRNA}

In accordance with the manufacturer's instructions, TLR4 siRNA or scrambled siRNA $(5 \mu \mathrm{g})$ was mixed with $40 \mu \mathrm{l}$ of transfection reagent NANOPARTICLE (Altogen Biosystems, Las Vegas, NV, USA) in a total volume of $100 \mu \mathrm{l}$ of $5 \%$ glucose $(\mathrm{w} / \mathrm{v})$. Then, 30 STZ-induced hyperglycemic C57BL/6 mice were randomly assigned into three groups of ten mice. The STZinduced hyperglycemic mice were untreated or intravenously injected with the TLR4 siRNA or scrambled mixture via the tail vein. In addition, ten non-diabetic mice were used as control. Four weeks after the first treatment, the diabetic mice were injected again with the same dose of the TLR4 siRNA or scrambled siRNA.

\section{Histological Analysis}

Hearts were excised from the mice and washed with saline solution. They were then fixed with $10 \%$ formalin. To observe the left and right ventricle, hearts were cut transversely. To detect collagen deposition, heart sections with a thickness of $5 \mu \mathrm{m}$ were stained with hematoxylin and eosin (H\&E) and a saturated solution of picric acid containing 1\% Sirius red. Thes sections were then observed under light microscopy using computer-assisted 
morphometry (Image-Pro Plus Version 6.0). All available fields (more than 30 fields) were measured in each sample, including the septum, the right ventricles, and the left ventricles (all fields were analyzed using a $\times 40$ objective lens). For the cross-sectional area of cardiomyocytes, sections were stained with fluorescein isothiocyanate (FITC)-conjugated wheat germ agglutinin (WGA; Invitrogen) to detect membranes and with 4,6-diamidino-2-phenylindole (DAPI) to detect nuclei. Individual cardiomyocytes were measured with a quantitative digital image analysis system (NIH Image version 1.6). The outline of 200 cardiomyocytes was traced in each section.

\section{Quantitative Real-Time Polymerase Chain Reaction (Q-RT-PCR)}

Total RNA was extracted from heart tissues through Trizol reagent (Gibco-BRL) according to the manufacturer's instruction. RNA was then reverse-transcribed using an oligo-(dT) primer and reverse transcriptase (Invitrogen). Quantitative real-time polymerase chain reaction (Q-RT-PCR) was performed to analyze mRNA expression for beta-myosin heavy chain $(\beta-\mathrm{MHC})$, atrial natriuretic peptide (ANP), caspase-3, collagen I and III (Col I and III), transforming growth factor- $\beta 1$ (TGF $\beta 1$ ), interferon alpha (INF $\alpha$ ), intercellular cell adhesion molecule 1 (ICAM-1), vascular cell adhesion molecule 1 (VCAM-1), interleukin-1 $\beta$ (IL-1 $\beta$ ), tumor necrosis factor- $\alpha$ (TNF $\alpha$ ), and glyceraldehyde-3-phosphate dehydrogenase (GAPDH) as previously described. The primers used are as follows.

ANP: $\quad$ 5'-GGCTCCTTCTCCATCACCAA-3' (forward) and 5'-CGAGAGCACCTCCATCTCTC$3^{\prime}$ (reverse).

$\beta$-MHC: $\quad$ '-GTCAAGCTCCTAAGTAATCTG TT-3' (forward) and 5'-GAAAGGATGAGCCTT TCTTTGC-3' (reverse).

TGFß1: 5 '-AAGAAGTCACCCGCGTGCTA-3' (forward) and 5'-TGTGTGATGTCTTGGTT TTGTCA-3' (reverse).

Col I: 5-TCCTGGCAACAAAGGAGACA-3 (forward) and 5-GGGCTCCTGGTTTTCCTTCT3 (reverse).
Col III: 5-ACGTAGATGAATTGGGATGCAG3 (forward) and 5-GGGTTGGGGCAGTCTAG TC-3 (reverse).

VCAM-1: 5-TACCAGCTCCCAAAATCCTG-3 (forward) and 5-TCTGCTAATTCCAGCCTCGT-3 (reverse).

ICAM-1: 5-GTGATCCCTGGGCCTGGTG-3 (forward) and 5-GGAAACGAATACACGGT GATGG-3 (reverse).

TNFa: 5-CAGCCGATGGGTTGTACCTT-3 (forward) and 5-TGTGGGTGAGGAGCACGTA GT-3 (reverse).

IL-1 1 : 5-CAGTTCTGCCATTGACCATC-3 (forward) and 5-TCTCACTGAAACTCAGCCGT3 (reverse).

GAPDH: 5'-ACTCCACTCACGGCAAATTC$3^{\prime}$ (forward) and 5'-TCTCCATGGTGGTGAAG ACA-3' (reverse).

The Q-RT-PCR reaction conditions were $95^{\circ} \mathrm{C}$ for $10 \mathrm{~min}, 95^{\circ} \mathrm{C}$ for $30 \mathrm{~s}, 58^{\circ} \mathrm{C}$ for $1 \mathrm{~min}$, and $72^{\circ} \mathrm{C}$ for $30 \mathrm{~s}$ (40 cycles). More details of Q-RT-PCR reactions were reported in our previous study [15]. Data were analyzed using MX4000 (Stratagene), Microsoft Excel2010, and GraphPad Prism software.

\section{Echocardiography}

The mice were anesthetized by intraperitoneal injection of ketamine $(120 \mathrm{mg} / \mathrm{kg})$. Body temperature was maintained at $36.5-37.5^{\circ} \mathrm{C}$. Mice were imaged in a warm handing platform using a $40-\mathrm{MHz}$ linear array transducer attached to a cardiovascular ultrasound system (Vivo 2100, Visual Sonics). M-mode and 2-D parasternal short-axis scans at the level of the papillary muscles were used to assess changes in left ventricle fractional shortening (FS) and ejection fraction $(\mathrm{EF})$.

\section{Statistical Analysis}

All data are expressed as the mean \pm SE. Oneway ANOVA with Newman Keuls test was used to compare two groups. Statistical analyses were conducted through SPSS 25.0 (IBM Corp., Armonk, NY, USA). Differences were considered to be significant at a two-tailed $p<0.05$. 


\section{RESULTS}

\section{Anti-hypertrophic Effects of TLR4 siRNA in Hearts of Diabetic Mice}

Diabetes can induce cardiac hypertrophy, one of the most representative changes of diabetic cardiomyopathy. Cardiomyocyte cross-sectional areas were significantly increased in hearts of diabetic mice compared with those of non-diabetic mice, indicating hypertrophy in the former. To test the anti-hypertrophic effect of TLR4 siRNA, we first knocked down the expression of TLR4 using gene silencing technology. The results showed that the TLR4 mRNA level was decreased by $72 \%$, as compared with the mice treated with scrambled control siRNA after infusion of TLR4 siRNA, indicative of successful knockdown in the heart in vivo (Fig. 1a). Knockdown of TLR4 decreased cardiomyocyte cross-sectional areas in hearts of STZ-treated diabetic mice (Fig. 1b, c). Similarly, the induction of cardiac fetal gene expression of ANP and $\beta$-MHC (Fig. 1d, e), two markers of cardiac hypertrophy, was significantly reduced in TLR4 siRNA-treated hearts in response to diabetes. Moreover, the levels of random glucose in STZ-induced diabetic mice $(20.49 \pm 1.63)$ were higher than those in normal mice $(11.56 \pm 1.72)(p<0.05)$. However, the levels of random glucose in TLR4 siRNAtreated mice with STZ-induced hyperglycemia $(18.86 \pm 1.45)$ were similar to those the scrambled siRNA group $(20.42 \pm 1.68) \quad(p>0.05)$ (Fig. 1f). These results indicate that silencing TLR4 can not lower the blood glucose but can prevent the development of cardiac hypertrophy in diabetic mice.

\section{Inhibitory Effects of TLR4 siRNA on Myocardial Fibrosis in Diabetic Mice}

To delineate the role of TLR4 in myocardial fibrosis, at first we analyzed total collagen contents in the diabetic mouse hearts. Initially, we found that collagen deposition significantly increased in diabetic mouse hearts, whereas siRNA-mediated silencing of TLR4 reduced diabetes-induced collagen deposition (Fig. 2a, b).
Similar to the total collagen contents, the mRNA levels of collagens I and III in hearts from TLR4 siRNA-treated mice were much lower than that of the control siRNA-treated diabetic mice (Fig. 2d, e).

To gain further insight into the role of TLR4 in diabetes-induced fibrosis, we analyzed the expression of the pro-fibrotic gene TGF $\beta 1$ in the diabetic mouse hearts. In non-diabetic mice, there was no difference in the TGF $\beta 1$ mRNA levels between TLR4 siRNA- and control siRNAtreated hearts. However, the TGF $\beta 1$ mRNA levels were significantly upregulated in control siRNA-treated hearts in response to STZ (Fig. 2c). In parallel to the changes in collagen deposition and collagen I and III expression, knockdown of TLR4 reduced TGF $\beta 1$ mRNA expression in the hearts of diabetic mice.

\section{Reduced Inflammatory Response in Hearts of TLR4 siRNA-Treated Diabetic Mice}

Inflammation has been suggested to play an important role in the development of myocardial remodeling [8]. Therefore, we analyzed proinflammatory cytokine expression in the hearts of diabetic mice. Indeed, in diabetic mice, significantly increased levels of TNF $\alpha$ and IL-1 were detected (Fig. 3a, b). Administration of TLR4 siRNA prevented this increase in cytokine expression.

In parallel experiments, we also examined adhesion molecule expression in hearts of diabetic mice. Diabetes induced the upregulation of ICAM- 1 and VCAM-1 expression in the hearts of diabetic mice (Fig. 3c, d). This increased expression of ICAM-1 and VCAM-1 was significantly attenuated by TLR4 siRNA treatment. These results suggest that knockdown of TLR4 prevents the myocardial inflammatory response to diabetes.

\section{Attenuation of Myocardial Dysfunction in TLR4 siRNA-Treated Diabetic Mice}

Having shown that silencing TLR4 prevents apoptosis, hypertrophy, fibrosis, and inflammation in hearts of diabetic mice, each of which contributes to diabetic cardiomyopathy, we 
A

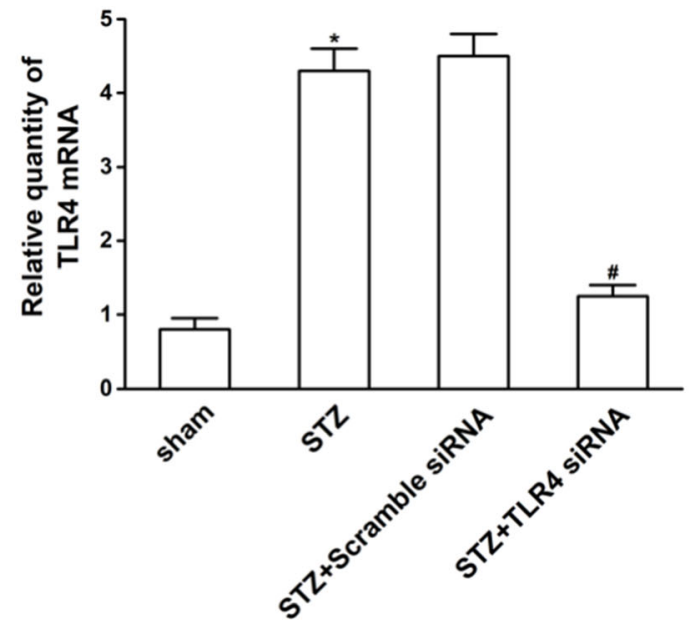

C

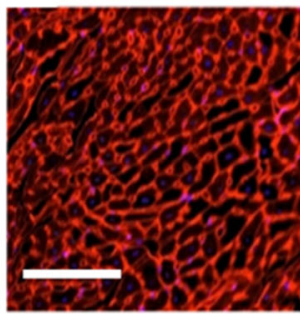

Sham

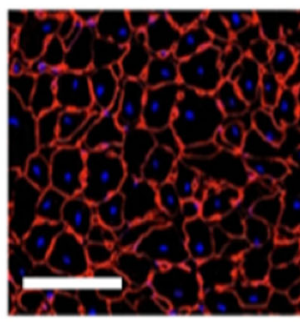

Scramble siRNA

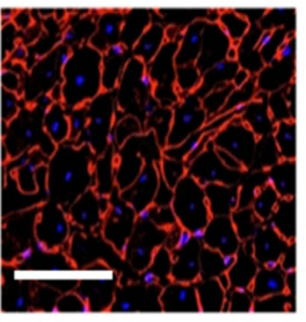

STZ

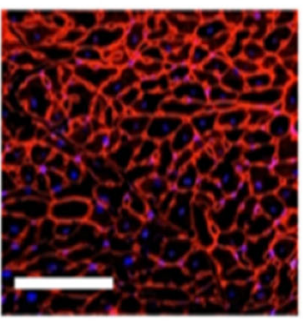

TLR4 SIRNA

E

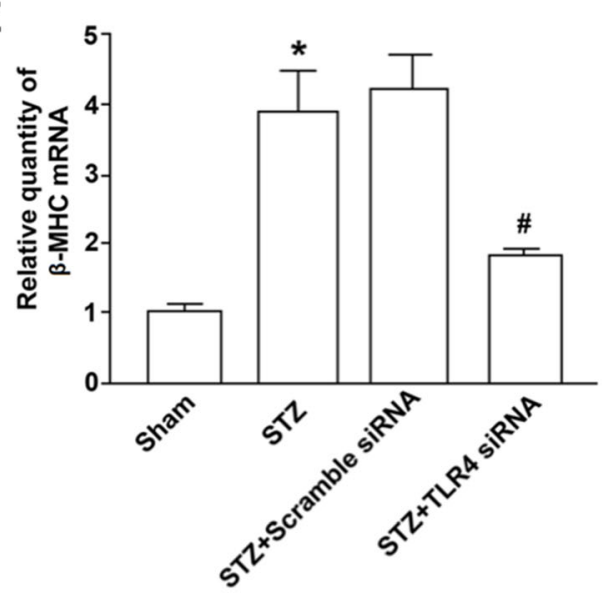

B

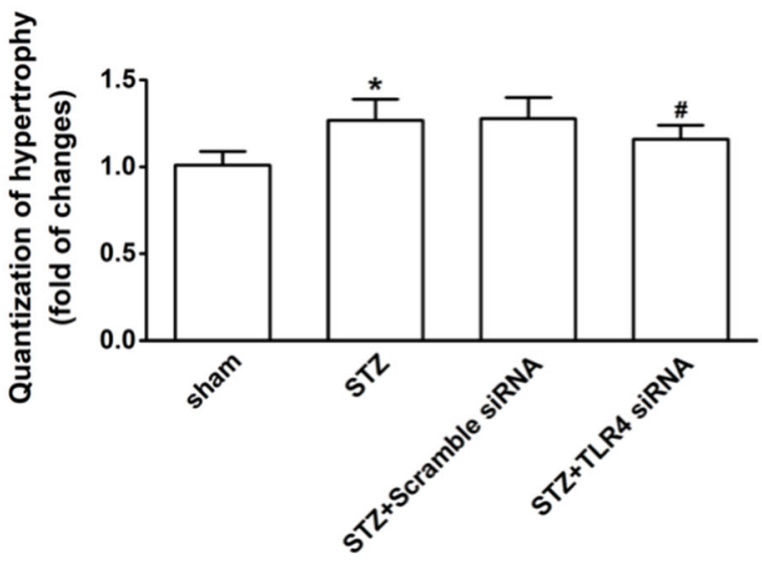

D

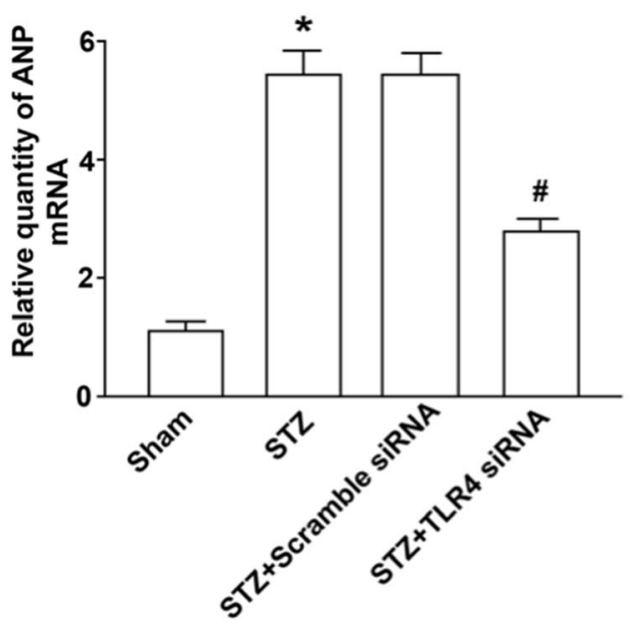

$\mathbf{F}$

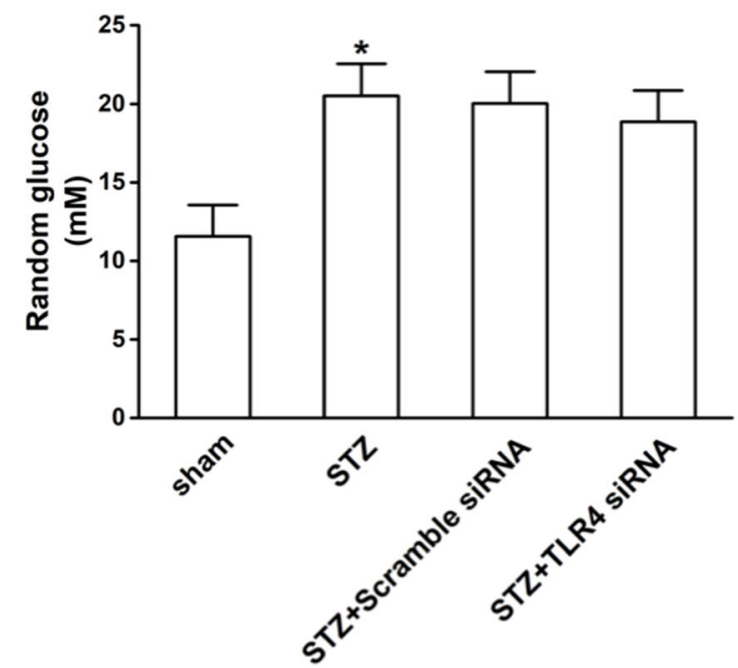


4Fig. 1 TLR4 siRNA displayed anti-hypertrophic effort in hearts of STZ-induced diabetic mice. Four experimental groups included the untreated mice (sham), diabetic mice (STZ), and the mice treated with scrambled siRNA and TLR4 siRNA. Each group contained ten mice. Eight weeks after treatment, hearts were excised, fixed, and sectioned. Total mRNA was extracted and used to detect the TLR4 transcripts by Q-RT-PCR. The relative quantity of TLR4 mRNA was expressed as mean \pm SD. a TLR4 expression in the hearts of mice. TLR 4 mRNA level was decreased by $72 \%$, as compared with the mice treated with scrambled control siRNA after infusion of TLR4 siRNA. b Quantification of hypertrophic cells. To determine cardiomyocyte cross-sectional area, sections were stained with FITC-conjugated wheat germ agglutinin to detect membranes. Single cardiomyocytes in the sections were measured with a quantitative digital image analysis system. The outline of 200 cardiomyocytes was traced in each section. The increase of cell sizes was quantified as compared with the cardiomyocyte of normal mice (sham). c Representative fluorescence images of the hypertrophic cells ( $\times 200)$. The scale bars are $50 \mu \mathrm{m}$. d, e Compared to the controls, the induction of cardiac fetal gene expression of ANP and $\beta$-MHC were reduced in TLR4 siRNAtreated hearts. $\mathbf{f}$ The levels of random glucose in TLR4 siRNA-treated mice with STZ-induced hyperglycemia were similar to those in the scrambled siRNA group $(p>0.05) .{ }^{\#}$ Statistical significance when compared with scrambled siRNA-treated mice, or ${ }^{*}$ with untreated mice (sham) with STZ-induced mice were denoted as $p<0.05$

hypothesized that the knockdown of TLR4 would attenuate myocardial dysfunction in diabetic mice. To examine this hypothesis, we measured myocardial function in diabetic mice using echocardiography. Compared with hearts of non-diabetic mice, diabetic mice showed decreases in left ventricle FS $(27.33 \% \pm 3.20 \%$ vs. $35.33 \% \pm 1.74 \%, p<0.05)$ and $\mathrm{EF}$ values $(48.26 \% \pm 5.21 \% \quad$ vs. $\quad 67.18 \% \pm 4.73 \%$, $p<0.05)$, indicative of myocardial dysfunction (Fig. $4 \mathrm{a}-\mathrm{c}$ ). By contrast, both FS and EF values were preserved in TLR4 siRNA-treated diabetic mice compared with control siRNA-treated ones $(31.80 \% \pm 2.82 \%$ vs. $28.50 \% \pm 5.83 \%$ for $\mathrm{FS}$ $p<0.05)(57.95 \% \pm 6.48 \%$ vs. $45.34 \% \pm 4.25 \%$ for EF $p<0.05)$. TLR4 siRNA treatment in nondiabetic animals did not affect myocardial function, indicating no potential side effects of TLR4 siRNA or nanoparticle-based transfection reagents. Thus, knockdown of TLR4 protects myocardial function in diabetic mice. Meanwhile, compared with control siRNA-treated diabetic mice, the TLR4 siRNA-treated mice had much smaller left ventricular volumes at diastolic phase $(50.55 \mu \mathrm{l} \pm 17.24 \mu \mathrm{l} \quad$ vs. $66.38 \mu \mathrm{l} \pm 14.16 \mu \mathrm{l}, p<0.05)$ and at systolic phase $(17.50 \mu \mathrm{l} \pm 8.77 \mu \mathrm{l}$ vs. $36.67 \mu \mathrm{l} \pm 9.98 \mu \mathrm{l}$, $p<0.05$ ) (Fig. $4 \mathrm{~d}, \mathrm{e}$ ), implying that TLR4 knockdown could ameliorate the development of myocardial remodeling.

\section{DISCUSSION}

Diabetic cardiomyopathy, one of the most common complications in patients with diabetes mellitus, is defined as ventricular dysfunction independent of hypertension and coronary artery disease [19-25]. At present, the morbidity of diabetic cardiomyopathy is gradually increasing with the growing number of individuals with diabetes [9]. Although the mechanism of diabetic cardiomyopathy is poorly understood, hypertrophy, fibrosis, and the inflammation of cardiomyocytes are reported as the predominant major factors causing myocardial dysfunction [6]. Moreover, diabetes increases hypertrophy, fibrosis, and inflammation of cardiomyocytes in diabetic animals and patients $[6,7,26]$. TLRs are the proximal signaling receptors in innate immune responses. Among the 10 identified human TLRs, at least two of them, TLR2 and TLR4, exist abundantly in the heart $[27,28]$. However, the role of TLR4 in myocardial dysfunction has not been characterized. We also found that TLR4 plays a crucial role in cardiac apoptosis. The level of TLR4 mRNA was significantly elevated in myocardial tissue after 7 days of hyperglycemia. However, after TLR4 siRNA treatment, cell apoptosis in myocardial tissues was attenuated significantly, which was associated with caspase-3 inactivation [14]. In this study, we provide more evidence that hyperglycemia is capable of triggering hypertrophy, fibrosis, and cardiomyocytic inflammation. Furthermore, we demonstrate that TLR4 expression in the myocardia of STZ-treated mice is significantly increased. The hypertrophy, fibrosis, 
A

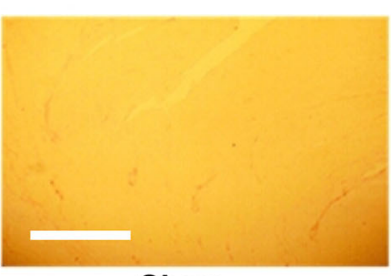

Sham

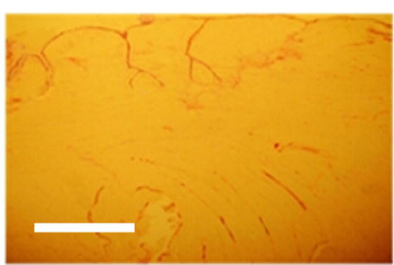

Scrambled siRNA

C

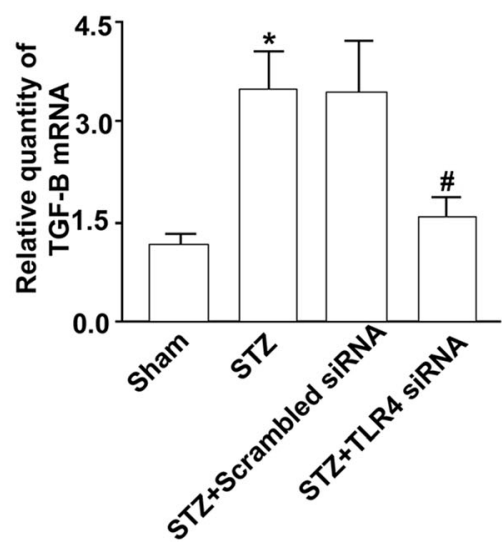

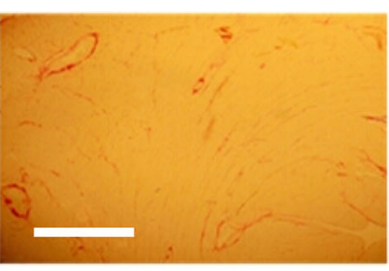

STZ

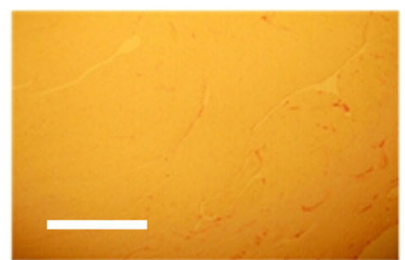

TLR4 SIRNA

D

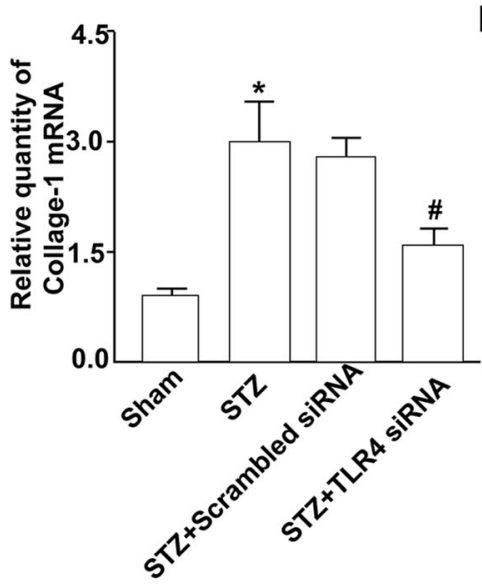

B

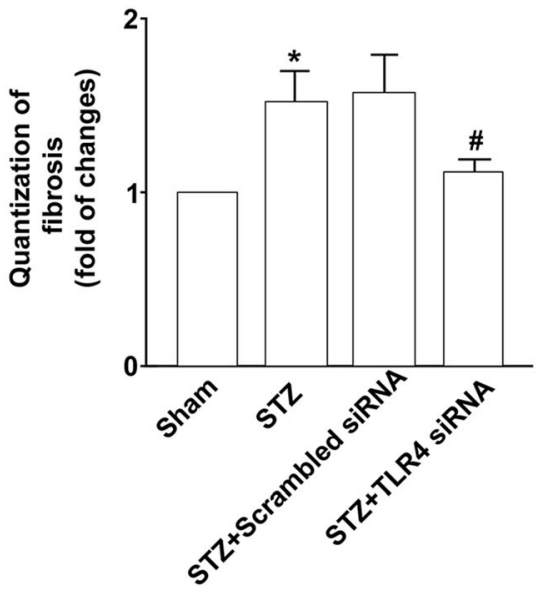

E

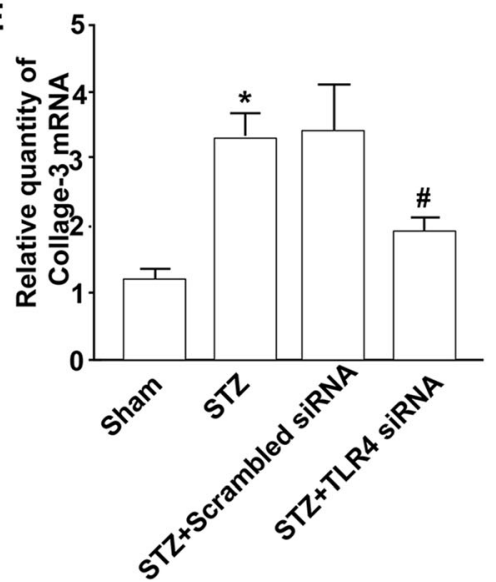

Fig. 2 Inhibition of myocardial fibrosis by TLR4 siRNA. Diabetic mice were made and treated with TLR 4 siRNA as described in this figure. Four experimental groups included the untreated mice (sham), diabetic mice (STZ), and the mice treated with scrambled siRNA and TLR4 siRNA. Eight weeks after treatment, hearts were excised, fixed, and sectioned. a The sections were stained with H\&E and a saturated solution of picric acid containing 1\% Sirius red for collagen deposition. The sections were then visualized by light microscopy, photographed $(\times 100)$. The scale bars are $100 \mu \mathrm{m}$. b The collagen content of the sections was measured by using the computer-assisted morphometry.

inflammation of cardiomyocytes, and myocardial dysfunction can be attenuated by knockdown of the TLR4 gene.

Hypertrophy of cardiomyocytes is one of the features of diabetic cardiomyopathy. c TGF $\beta 1$ expression in myocardia. mRNA from hearts from four experimental groups of mice were prepared for detecting TGF $\beta 1$ using Q-RT-PCR as described in "Methods". d, e Collagen I and collagen III expression in myocardia. mRNA from hearts from four experimental groups of mice were prepared for detecting collagen 1 using Q-RT-PCR. Data shown are representative of three experiments. \#Statistical significance when compared with scrambled siRNA-treated mice, or ${ }^{*}$ with untreated mice (sham) with STZ-induced diabetic mice were denoted as $p<0.05$

Fibroblasts, a type of cardiac cell, were present in significantly higher numbers in myocardial tissue of diabetic cardiomyopathy and could further induce the synthesis and metabolism of collagen. Type I and type III collagens are the 
A

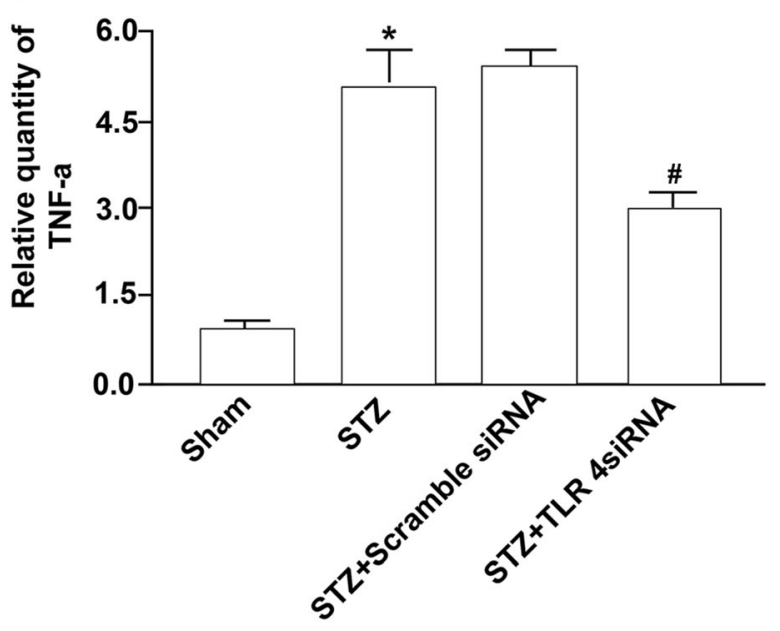

C

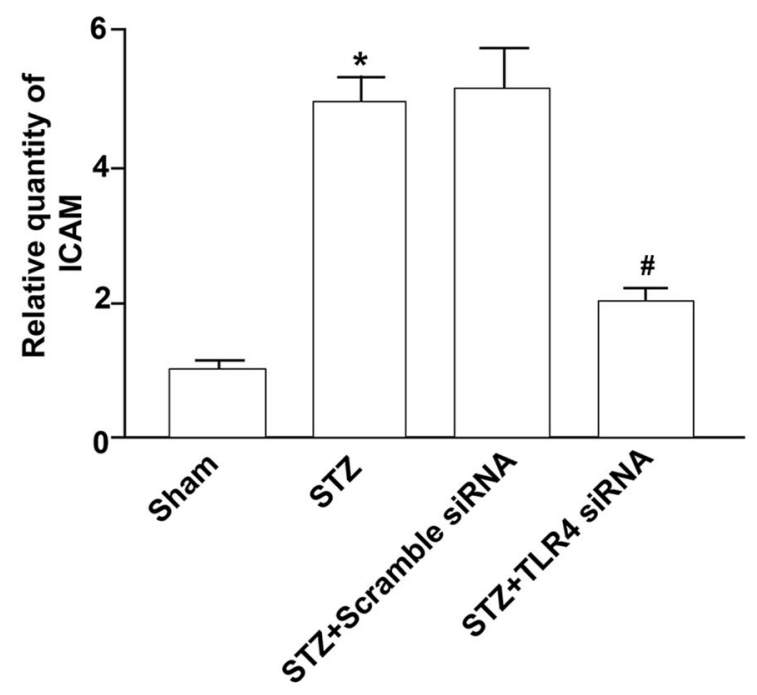

Fig. 3 Suppression of inflammatory response by TLR4 siRNA. Diabetic mice were made and treated with TLR4 siRNA as described before. Four experimental groups included the untreated mice (sham), diabetic mice (STZinduced), and the mice treated with scrambled siRNA and TLR4 siRNA. Eight weeks after treatment, mRNA from hearts from four experimental groups of mice were

main components of the extracellular matrix. Increased collagen in myocardial tissue can lead to a decrease of myocardial compliance and ventricular elasticity in diabetic mice. Visually, the disorders and changes of collagens I and III in the fibroblast hypertrophy of diabetic cardiomyopathy were the major factors that
B

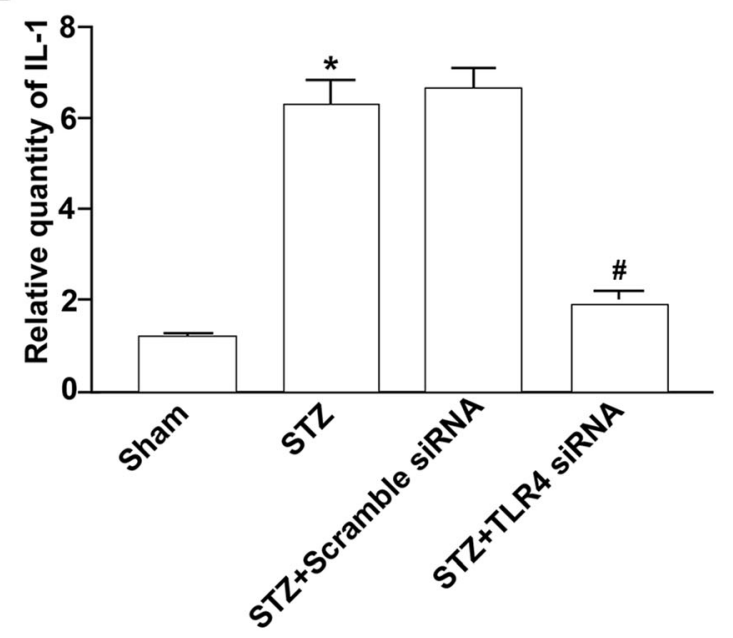

D

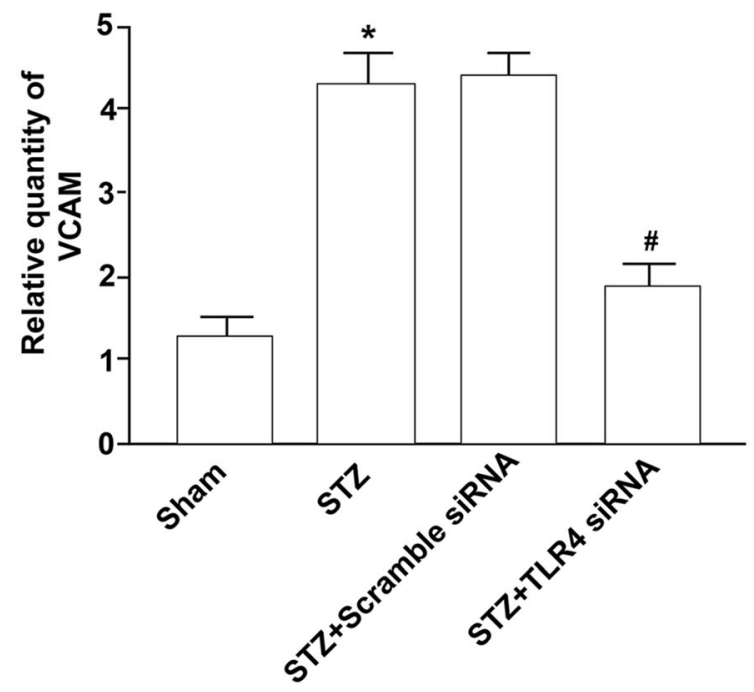

prepared for detecting TNF $\alpha(\mathbf{a})$, IL-1 (b), ICAM-1 (c), and VCAM-1 (d) using Q-RT-PCR as described in Fig. 1. Data shown are representative of three experiments. ${ }^{\#}$ Statistical significance when compared with scrambled siRNA-treated mice, or ${ }^{*}$ with untreated mice (sham) with STZ-induced diabetic mice were denoted as $p<0.05$

caused the accumulation of extracellular matrix and cardiac diastolic dysfunction. In our study, we showed that TLR4 siRNA reduced the myocardial fibrosis in diabetic mice.

ANP is a hormone synthesized and secreted by the cardiac tissue. Structural and functional changes in the heart will cause disorders of ANP 
A

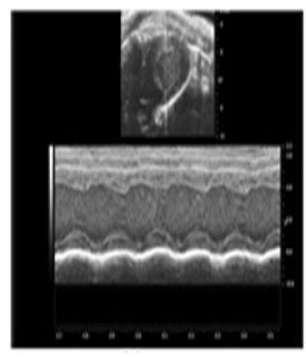

Sham

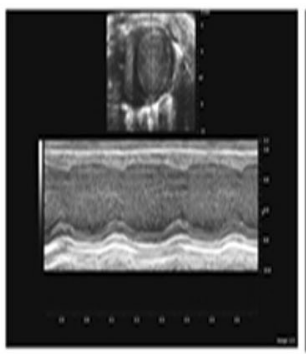

Scrambled siRNA

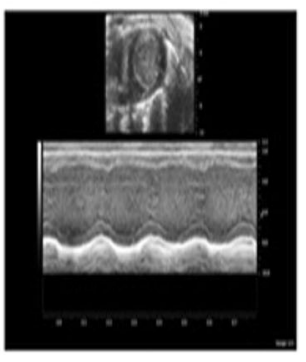

STZ

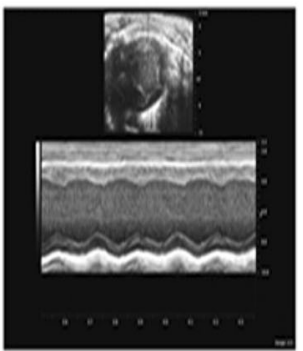

TLR4 SiRNA
B

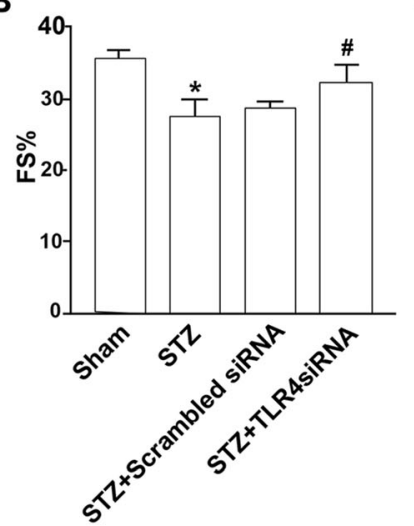

D

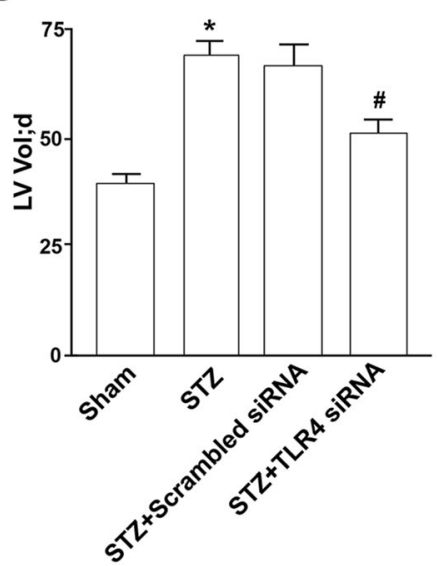

C

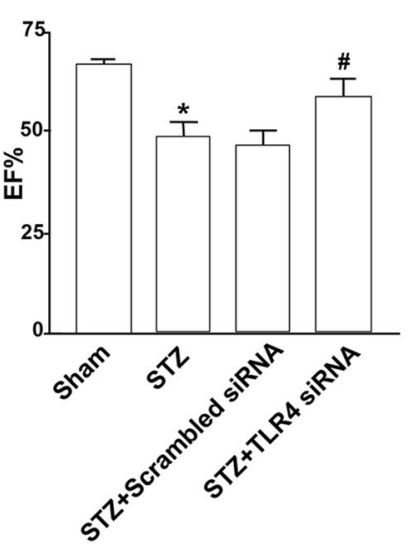

E

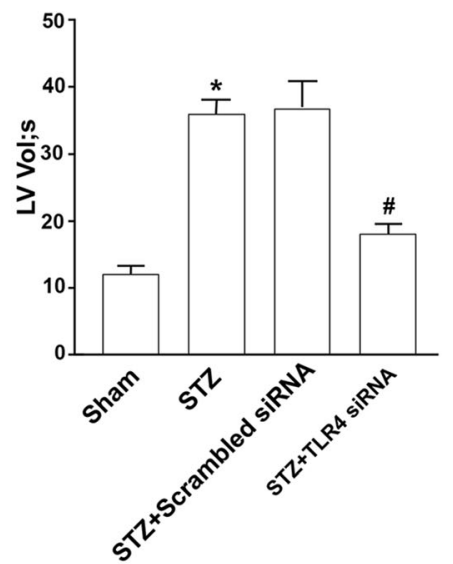

Fig. 4 Attenuation of myocardial dysfunction by TLR 4 siRNA. Diabetic mice were made and treated with TLR4 siRNA as described. Four experimental groups included the untreated mice (sham), diabetic mice (STZ), and the mice treated with scrambled siRNA or TLR4 siRNA. a Echocardiography. Eight weeks after treatment, the hearts were imaged by a cardiovascular ultrasound system. M-mode and 2-D parasternal short-axis scans at the levels of the papillary muscles were used to assess changes in left ventricle fractional shortening (FS) (b) and ejection

secretion. There is a significant relationship between ANP secretion and myocardial hypertrophy. Our study showed that silencing TLR4 prevented the development of cardiac hypertrophy in diabetic mice.

Studies have shown that inflammation has a major effect on diabetic cardiomyopathy. TNF $\alpha$ antagonism attenuates the development of diabetic cardiomyopathy associated with a reduction of intramyocardial inflammation and cardiac fibrosis. Cardiac cytokines, such as IL-1 fraction $(\mathrm{EF})$ (c). d, e The left ventricle volumes at diastolic or systolic phase were much smaller in TLR4 siRNA-treated mice than in STZ-treated or scrambled siRNA-treated mice. Data shown are representative of three experiments. ${ }^{\#}$ Statistical significance when compared with scrambled siRNA-treated mice, or ${ }^{*}$ with untreated mice (sham) with STZ-induced diabetic mice was denoted as $p<0.05$

and TGF $\beta$, are involved in the development of cardiac fibrosis and heart failure. $\beta$-MHC is associated with chronic hyperglycemia-induced oxidative stress. New methods are being developed to prevent diabetic cardiomyopathy by blocking inflammation associated with these factors. There is a close relationship between TLR4 expression and inflammation. Our study revealed that silencing TLR4 prevented the development of inflammation in diabetic mice. 
New studies suggest that diabetes is related to cardiomyocyte dysfunction. Moreover, the morbidity of diabetic cardiomyopathy is increasing $[29,30]$. However, the causes of diabetic cardiomyopathy are unknown, and effective methods to treat this disease are lacking. To clarify the role of TLR4 played in diabetic cardiomyopathy, we silenced the TLR4 gene using siRNA. Our study showed that TLR4 expression is related to myocardium trauma in STZ-induced diabetic mice. Silencing TLR4 can prevent hypertrophy, fibrosis, and inflammation in myocardial hearts to improve heart function. These results are consistent with previous studies [31-33].

Much research about TLR4 has been published in recent years mainly addressing its association with inflammation, sepsis, cancer, obesity, and atherosclerosis [34-38]. This article and our previous research highlight its effect on diabetic cardiomyopathy, an important potentially fatal complication of diabetes, possibly through upregulation of IFN and TNF and inducement of NADPH oxidase activation and ROS production in cardiomyocytes, a different mechanistic pathway compared with previous research. Moreover strong evidence has been provided about TLR4 accessory proteins MD2 and myocardial inflammatory injuries which sheds new light on our future research direction [28].

siRNA could be designed to target and silence virtually all mRNA. However, the major obstacle in clinical application of siRNA lies in developing safe and effective methods of delivering siRNA to target cells. siRNA requires specific vehicles that could facilitate its intracellular uptake and cytosolic delivery for bioactivity. In a later study, we have attempted to develop tissue- and cell-specific siRNA delivery carriers.

\section{CONCLUSION}

We explored the potential therapeutic reduction of apoptosis through the use of siRNA. Using siRNA to silence specific genes is the cutting edge of RNA interference, showing its potency and specificity in knocking down gene expression compared with traditional gene expression blocking methods. Our study revealed that the use of TLR4 siRNA can prevent hypertrophy, fibrosis, and cardiomyocytic inflammation in STZ-induced diabetic mice, thus highlighting the potential clinical application of siRNA-based therapy.

\section{ACKNOWLEDGEMENTS}

Funding. This study and rapid service fee was supported by the National Natural Science Foundation of China (Grant no. 81400665) and the Karamay projects for scientific and technological cooperation and exchange fund (2018HZ004A).

Authorship. All named authors meet the International Committee of Medical Journal Editors (ICMJE) criteria for authorship for this article, take responsibility for the integrity of the work as a whole, and have given their approval for this version to be published.

Authorship Contributions. YZ carried out the studies, participated in collecting and analyzing data, and drafted the manuscript. LT, SX, and YL performed the statistical analysis and data collection. FZ provided the experimental data and suggestions on experimental design. $\mathrm{XH}$ participated in data collecting and made revisions to the manuscript. NT and WM participated in the research proposal, project design, and manuscript writing.

Disclosures. Yuwei Zhang, Yang Li, Xuefang Huang, Fang Zhang, Lizhi Tang, Shishi Xu, Yuqi Liu, Nanwei Tong, and Weiping Min have nothing to disclose.

Compliance with Ethics Guidelines. All experimental procedures were approved by the Animal Use Subcommittee at Sichuan University.

Data Availability. The datasets used and/or analyzed during the current study are available from the corresponding author on reasonable request. 
Open Access. This article is licensed under a Creative Commons Attribution-NonCommercial 4.0 International License, which permits any non-commercial use, sharing, adaptation, distribution and reproduction in any medium or format, as long as you give appropriate credit to the original author(s) and the source, provide a link to the Creative Commons licence, and indicate if changes were made. The images or other third party material in this article are included in the article's Creative Commons licence, unless indicated otherwise in a credit line to the material. If material is not included in the article's Creative Commons licence and your intended use is not permitted by statutory regulation or exceeds the permitted use, you will need to obtain permission directly from the copyright holder. To view a copy of this licence, visit http://creativecommons.org/licenses/by$\mathrm{nc} / 4.0 /$.

\section{REFERENCES}

1. Rubler S, Dlugash J, Yuceoglu YZ, et al. New type of cardiomyopathy associated with diabetic glomerulosclerosis. Am J Cardiol. 1972;30:595-602.

2. Mazzone T, Chait A, Plutzky J. Cardiovascular disease risk in type 2 diabetes mellitus: insights from mechanistic studies. Lancet. 2008;371:1800-9.

3. Riad A, Bien S, Gratz M, et al. Toll-like receptor-4 deficiency attenuates doxorubicin-induced cardiomyopathy in mice. Eur J Heart Fail. 2008;10: 233-43.

4. De Groote P, Lamblin N, Mouquet F, et al. Impact of diabetes mellitus on long-term survival in patients with congestive heart failure. Eur Heart J. 2004;25: 656-62.

5. Feng Q, Song W, Lu X, et al. Development of heart failure and congenital septal defects in mice lacking endothelial nitric oxide synthase. Circulation. 2002;106:873-9.

6. Ares-Carrasco S, Picatoste B, Benito-Martin A, et al. Myocardial fibrosis and apoptosis, but not inflammation, are present in long-term experimental diabetes. Am J Physiol Heart Circ Physiol. 2009;297: H2109-19.
7. Sorrentino R, Arditi M. Innate immunity, Toll-like receptors, and atherosclerosis: mouse models and methods. Methods Mol Biol. 2009;517:381-99.

8. Hayat SA, Patel B, Khattar RS, et al. Diabetic cardiomyopathy: mechanisms, diagnosis and treatment. Clin Sci (Lond). 2004;107:539-57.

9. Fang ZY, Prins JB, Marwick TH. Diabetic cardiomyopathy: evidence, mechanisms, and therapeutic implications. Endocr Rev. 2004;25:543-67.

10. Zhao P, Wang J, He L, et al. Deficiency in TLR4 signal transduction ameliorates cardiac injury and cardiomyocyte contractile dysfunction during ischemia. J Cell Mol Med. 2009;13:1513-25.

11. Schnare M, Barton GM, Holt AC, et al. Toll-like receptors control activation of adaptive immune responses. Nat Immunol. 2001;2:947-50.

12. Goldstein DR. Toll-like receptors and other links between innate and acquired alloimmunity. Curr Opin Immunol. 2004;16:538-44.

13. Takeda K, Akira S. Toll-like receptors in innate immunity. Int Immunol. 2005;17:1-14.

14. Cai L, Kang YJ. Cell death and diabetic cardiomyopathy. Cardiovasc Toxicol. 2003;3:219-28.

15. Zhang Y, Peng T, Zhu $\mathrm{H}$, et al. Prevention of hyperglycemia-induced myocardial apoptosis by gene silencing of Toll-like receptor-4. J Transl Med. 2010;8:133.

16. Poucher SM, Cheetham S, Francis J, et al. Effects of saxagliptin and sitagliptin on glycaemic control and pancreatic beta-cell mass in a streptozotocininduced mouse model of type 2 diabetes. Diabetes Obes Metab. 2012;14:918-26.

17. Luo J, Quan J, Tsai J, et al. Nongenetic mouse models of non-insulin-dependent diabetes mellitus. Metab Clin Exp. 1998;47:663-8.

18. Wang HJ, Kang PF, Wu WJ, et al. Changes in cardiac mitochondrial aldehyde dehydrogenase 2 activity in relation to oxidative stress and inflammatory injury in diabetic rats. Mol Med Rep. 2013;8: 686-90.

19. Deedwania P, Kosiborod M, Barrett E, et al. Hyperglycemia and acute coronary syndrome: a scientific statement from the American Heart Association Diabetes Committee of the Council on Nutrition, Physical Activity, and Metabolism. Circulation. 2008;117:1610-9.

20. Adeghate E. Molecular and cellular basis of the aetiology and management of diabetic 
cardiomyopathy: a short review. Mol Cell Biochem. 2004;261:187-91.

21. Frustaci A, Kajstura J, Chimenti C, et al. Myocardial cell death in human diabetes. Circ Res. 2000;87: 1123-32.

22. Li Y, Li Y, Feng Q, et al. Calpain activation contributes to hyperglycaemia-induced apoptosis in cardiomyocytes. Cardiovasc Res. 2009;84:100-10.

23. Poornima IG, Parikh P, Shannon RP. Diabetic cardiomyopathy: the search for a unifying hypothesis. Circ Res. 2006;98:596-605.

24. Fiordaliso F, Bianchi R, Staszewsky L, et al. Antioxidant treatment attenuates hyperglycemia-induced cardiomyocyte death in rats. J Mol Cell Cardiol. 2004;37:959-68.

25. Chao W. Toll-like receptor signaling: a critical modulator of cell survival and ischemic injury in the heart. Am J Physiol Heart Circ Physiol. 2009;296:H1-12.

26. Zhu X, Zhao H, Graveline AR, et al. MyD88 and NOS2 are essential for toll-like receptor 4-mediated survival effect in cardiomyocytes. Am J Physiol Heart Circ Physiol. 2006;291:H1900-9.

27. Frantz S, Ertl G, Bauersachs J. Toll-like receptor signaling in the ischemic heart. Front Biosci. 2008;13:5772-9.

28. Baumgarten G, Knuefermann P, Nozaki N, et al. In vivo expression of proinflammatory mediators in the adult heart after endotoxin administration: the role of toll-like receptor-4. J Infect Dis. 2001;183: 1617-24.

29. Nathan DM, Genuth S, Lachin J, et al. The effect of intensive treatment of diabetes on the development and progression of long-term complications in insulin-dependent diabetes mellitus. N Engl J Med. 1993;329:977-86.

30. Miettinen H, Lehto S, Salomaa V, et al. Impact of diabetes on mortality after the first myocardial infarction. The FINMONICA Myocardial Infarction Register Study Group. Diabetes Care. 1998;21: 69-75.

31. Dinh W, Futh R, Nickl W, et al. Elevated plasma levels of TNF-alpha and interleukin-6 in patients with diastolic dysfunction and glucose metabolism disorders. Cardiovasc Diabetol. 2009;8:58.

32. Wu K, You Q, Mo HL, et al. Expression of Toll-like receptor 4 and tumor necrosis factor-alpha on peripheral-blood mononuclear cells and their correlation with myocardial perfusion in patients with diabetic cardiomyopathy. Zhonghua Xin Xue Guan Bing Za Zhi. 2011;39:503-7.

33. Tao A, Song J, Lan T, et al. Cardiomyocyte-fibroblast interaction contributes to diabetic cardiomyopathy in mice: role of HMGB1/TLR4/IL-33 axis. Biochim Biophys Acta. 1852;2075-2085:2015.

34. Rogero MM, Calder PC. Obesity, inflammation, toll-like receptor 4 and fatty acids. Nutrients. 2018;10:432.

35. Zhang YY, Liu X, Zhang X, et al. Shikonin improve sepsis-induced lung injury via regulation of miRNA140-5p/TLR4-a vitro and vivo study. J Cell Biochem. 2020;121:2103-17.

36. Li X, Li H, Dong X, et al. Expression of NF-kappaB and TLR-4 is associated with the occurrence, progression and prognosis of esophageal squamous cell carcinoma. Int J Clin Exp Pathol. 2018;11:5850-9.

37. Lin HY, Shao-Wen W, Shen FC et al. Abrogation of Toll like receptor 4 (TLR4) mitigates obesity-induced oxidative stress, proinflammation, and insulin resistance through metabolic reprogramming of mitochondria in adipose tissue. Antioxid Redox Signal. 2020. https://doi.org/10.1089/ars.2019. 7737.

38. Li H, Jiao Y, Xie M. Paeoniflorin ameliorates atherosclerosis by suppressing TLR4-mediated NFkappaB activation. Inflammation. 2017;40: 2042-51. 